\title{
EL MÉTODO DE JAMES SPRADLEY EN LA INVESTIGACIÓN CUALITATIVA
}

\author{
THE METHOD OF JAMES SPRADLEY IN QUALITATIVE RESEARCH \\ O MÉTODO DA JAMES SPRADLEY NA INVESTIGAÇÃO QUALITATIVA
}

\author{
Norman Garrido \\ Universidad de Tarapacá. Iquique, Chile. \\ ngarrido@uta.cl \\ ORCID 0000-0003-4918-5387
}

DOI: https://doi.org/10.22235/ech.v6iEspecial.1449

Recibido: $15 / 06 / 2017$

Aceptado: 05/09/2017

\section{RESUMEN}

En el presente artículo se expone el Método de la Secuencia de la Investigación desarrollado por James Spradley. Este procedimiento figura entre uno de los enfoques globales más importantes en los estudios cualitativos. La estrategia de Spradley fue diseñada de forma didáctica para orientar los trabajos de investigadores noveles cuya principal técnica de recopilación de información es la entrevista etnográfica. El trabajo de campo convierte al observador en un instrumento de investigación. En este sentido, es necesario contar con recomendaciones procedimentales para el adecuado estudio de las personas en sus escenarios vitales: lugares de trabajo, barrios, hospitales, escuelas, entre muchos espacios donde los individuos desarrollan su cotidianidad. Las orientaciones básicas para la observación y tratamiento de la información se exponen a través de una serie de etapas. La información procede del estudio de fenómenos vinculados a diversas ramas de las ciencias sociales y de la salud que estudian a las personas en sus contextos de vida. Los pasos de este método van creando esquemas conceptuales que orientan la forma de realizar el análisis de la información, obtenida del corpus de las entrevistas. El investigador recibe sugerencias que lo sitúan constantemente entre el análisis de los datos y la recopilación de información en el trabajo de campo. La utilización de este tipo de estrategia repetitiva permite adecuar la observación fundamentada en las preguntas y objetivos del estudio que se realiza, y resulta de gran ayuda para la obtención de datos que guíen la investigación hacia resultados atinentes en el proceso de conocer, describir y obtener recomendaciones para la intervención en los contextos estudiados.

Palabras clave: Entrevistas como Asunto, Metodología, Investigación Cualitativa, Semántica.

\section{ABSTRACT}

This paper shows the Developmental Research Sequence Method developed by James Spradley. This procedure is among one of the most important global approaches in qualitative research. The strategy of Spradley was designed in a didactic way to guide the work of junior researchers whose main technique for the collection of data is the ethnographic interview. The fieldwork turns the observer into a research tool. Thus, there are necessary procedural recommendations for the proper study of the people in their life settings such as workplaces, neighborhoods, hospitals and schools, among others, spaces where individuals develop their daily lives. The basic orientations for the observation and the processing of the information are shown in a sequence of steps. The data comes from the study of phenomena, related to various branches of the social and health sciences, where people are studied in their life context. The steps in this method create frameworks that guide the way to perform the analysis of the information. Hence, the researcher receives suggestions that place him constantly between the analysis of data and the collection of information in the field work. The use of this type of recursive technique allows him to adapt the observation, based on the questions and objectives of the study that has been carried out. It is of great help for obtaining data to guide the research towards connected results in the process of knowing, describing and getting recommendations to intervene in the contexts studied.

Keywords: Interviews as Topic, Methodology, Qualitative Research, Semantics.

\section{RESUMO}

Este artigo expõe o Método da Sequência da Investigação desenvolvido por James Spradley. Este procedimento figura entre um dos enfoques globais más importantes nos estudos qualitativos. A estratégia de Spradley foi desenhada de forma didática para orientar os trabalhos de investigadores noveis que usam a entrevista etnográfica como principal técnica de recopilação de informação. O trabalho de campo torna o observador em um instrumento de investigação. Neste sentido, é necessário contar com recomendações procedimentais para o estudo adequado das pessoas nos seus cenários vitais: lugares de trabaIho, bairros, hospitais, escolas, além de muitos espaços onde 
os indivíduos se desenvolvem cotidianamente. As orientações básicas para a observação e o tratamento da informação são expostas através de uma série de etapas. A informação provém do estudo de fenômenos vinculados a diferentes áreas das ciências sociais e da saúde que estudam as pessoas nos seus contextos de vida. Os procedimentos deste método vão criando esquemas conceptuais que orientam a forma de realizar a análise da informação, obtida do corpus das entrevistas. $O$ investigador recebe sugestões que o colocam constantemente entre a análise dos dados e a recopilação de informação no trabalho de campo. $\mathrm{O}$ uso deste tipo de estratégia repetitiva permite adequar a observação fundamentada nas perguntas e objetivos do estudo que se está realizando, e é de grande ajuda para a obtenção de dados que guiam a investigação a resultados pertinentes no processo de conhecer, descrever e obter recomendações para a intervenção nos contextos estudados.

Palavras chave: Entrevistas como Assunto, Metodologia, Pesquisa Qualitativa, Semântica.

\section{INTRODUCCIÓN}

James Spradley fue antropólogo, docente del Macalester College. Es autor de una veintena de textos entre los que destacan "Observación Participante" y "Entrevista Etnográfica". Como especialista etnógrafo, Spradley se dedicó durante su carrera a desarrollar procedimientos para el diseño y ejecución de investigaciones etnográficas con base analítica en la etnosemántica, cuyo objeto es conocer los dilemas que plantea el estudio del léxico en un ejercicio constante de agrupación y contraste de la información obtenida, principalmente a través de la entrevista etnográfica. En el planteamiento de Spradley convergen el trabajo de campo, la entrevista y el análisis de la información, lo que en una dinámica constante hace necesario regresar al escenario para recopilar, otra vez, más información.

Spradley es el creador del "Método de la Secuencia de Desarrollo de la Investigación (en adelante MSDI) que figura entre los tres procedimientos globales de mayor tradición en los estudios cualitativos, junto a la inducción analítica y la comparación constante. EI MSDI visualiza el trabajo etnográfico como una secuencia, que tiene su origen en la idea de investigación y continúa a través de todo el desarrollo del proceso. La recurrencia entre el análisis de la información recopilada y la necesidad de retornar al escenario es una característica destacada en esta estrategia. Este proceso de retroalimentación va desarrollándose hasta que, paulatinamente, el investigador obtenga resultados y conclusiones.

EI MSDI se centra en el trabajo etnográfico y su aplicación ha trascendido favorablemente hacia las ciencias de la salud, donde figura ya como parte del diseño metodológico de importantes investigaciones en las que se ha demostrado su aplicabilidad, tales como: lactancia materna de mujeres con diabetes, remedios tradicionales en la promoción de salud, vivencias de familiares en la sala de espera de establecimientos hospitalarios, entre otros (1 -3).

Es importante destacar que uno de los objetivos de Spradley fue que este método fuera también didáctico, para orientar el trabajo de investigadores iniciados en los procedimientos cualitativos, y de esta manera consiguió que fuera comprensible para el tratamiento de problemas en investigaciones culturales (4).

\section{LA ENTREVISTA ETNOGRÁFICA}

No hay consenso aún entre los investigadores en una definición de entrevista etnográfica que abarque todos los aspectos necesarios para esta técnica cualitativa. James Spradley alude a un recurso para hacer que la gente hable sobre lo que sabe (5). Para ello se precisa, necesariamente, la presencia de un entrevistado, un entrevistador y un tema alrededor del cual se produzca una interacción mediante preguntas y respuestas. El problema de determinar la ubicación de las entrevistas etnográficas en el abanico de técnicas cualitativas para recopilar información radica en su particular forma de desarrollo. Algunos la califican como un tipo de entrevista en profundidad, mientras que otros la diferencian de éstas por su singular forma de ser implementada, que va desde la no estructuración hasta la formulación de preguntas dirigidas durante las fases que contempla $(4,6)$. En el presente análisis situaremos esta técnica en una forma especial de entrevista cualitativa que se desarrolla en etapas en donde puede adquirir una o más de las características señaladas por Patton en su clasificación. Este autor distingue cuatro tipos de entrevistas:

- Entrevista conversacional informal: corresponde al estilo más abierto para desarrollar una entrevista, destacando la ausencia de temas y preguntas previas.

- Basada en un guión: su nombre señala su principal característica. Si bien el entrevistador posee la libertad para ordenar los momentos en que formula las interrogantes, debe observar la presencia de un guión preestablecido.

- $\quad$ Estandarizada abierta: se distingue por la presencia de un listado de preguntas redactadas y aplicadas por igual para todos los entrevistados, los que tienen libertad para responder.

- $\quad$ Estandarizada cerrada: se estructura en función de preguntas redactadas y formuladas por igual a todos los entrevistados, pero éstos no poseen libertad para responder, debiendo elegir entre alternativas acotadas (7).

Las entrevistas etnográficas adquieren, principalmente, las modalidades de las dos primeras, mientras la tercera se ubica en un punto intermedio entre las entrevistas cualitativas y los instrumentos propios de diseños cuantitativos. Las estandarizadas cerradas se reconocen como encuestas y cuestionarios.

La entrevista etnográfica es una conversación informal en la cual van surgiendo preguntas de forma natural, adaptada a los sujetos y las condiciones del contexto. Una importante característica de esta forma de realizar una entrevista radica en su flexibilidad. Los entrevistados tienen la libertad de explayar- 
se. Con todo, esta técnica no se desarrolla como conversación abierta; la necesidad de responder a preguntas de investigación induce al entrevistador a introducir estructuras o pautas en la interacción que le otorga el guion de temas a tratar $(7,8)$. Otra característica de este tipo de entrevistas es el tiempo que conllevan. Están vinculadas, por lo general, al trabajo de campo y a una serie de actividades conjuntas que se desarrollan en el escenario en donde transcurre el día a día de las personas seleccionadas como informantes. Las personas nos entregan información sobre sus vidas, de la que se intenta obtener datos sobre interacciones en torno al fenómeno de interés. Así, el investigador se convierte en un individuo que participa de las dinámicas de los individuos en su contexto, lo que se traduce no solo en realizar entrevistas, sino también observar ceremonias, juegos, tomar notas y aprender de las relaciones de las personas, todo lo que contempla un tiempo prudente en donde el investigador tiene la oportunidad de llevar a cabo entrevistas junto con otras técnicas de observación (5, 9-12).

La realización de una entrevista etnográfica no obedece a manuales de procedimiento encuadrados en rígidas estructuras; se relaciona más bien con el despliegue de habilidades comunicacionales que el investigador, convertido en el principal instrumento para recopilar información, es capaz de desarrollar $(5,6,13)$. Sin embargo, se observan una serie de fases, articuladas de forma secuencial, en un intento pedagógico de ofrecer recomendaciones que resultan sugerentes. De esta forma, se alude a una primera fase de preparación, en donde se cotejan aspectos organizativos: el establecimiento de objetivos, redacción de preguntas, definición de quiénes deben y pueden ser entrevistados, el momento y lugar para ejecutar la entrevista, la duración recomendada para las interacciones, tipo de registro, entre otros aspectos. Una segunda fase comienza con la interacción con el entrevistado en el lugar de la cita. Es el momento para solicitar la autorización para registrar el proceso. En este punto es importante destacar la posibilidad de utilizar dispositivos de registro digital de la voz debido a las ventajas que ofrecen. La captura del audio en las entrevistas se considera fundamental para la aplicación del MSDI con su elemental componente de análisis etnosemántico en el que la observación del discurso se convierte, junto con el registro de expresiones y modismos, en base para el desarrollo de esta estrategia. No obstante, la presencia de una grabadora digital puede convertirse en un elemento distractor, inhibidor, que perturbe la comunicación y dificulte estimular respuestas francas del entrevistado $(6,7,14)$. En la tarea de reducir el riesgo con la presencia de un dispositivo en el escenario, es necesario establecer vínculos de confianza previos con el informante en donde se aclaren dudas y se dé a conocer las intenciones de la investigación, sin descartar el ofrecimiento de anonimato. En una tercera fase, vinculada al pleno desarrollo de la entrevista, el entrevistador utiliza sus habilidades comunicacionales para mantener una conversación fluida, sin olvidar la guía o preguntas que se requiere responder. Con todo, se debe tener presente que el protagonista en el diálogo es el entrevistado, reduciendo la intervención del entrevistador a su papel de dinamizador de la conversación con sutiles formas de insertar las preguntas en el proceso. La siguiente y última fase corresponde al cierre de la entrevista; aquí el investigador, considerando el tiempo transcurrido y las respuestas obtenidas durante el desarrollo, estimula al entrevistado para que profundice temas a modo de síntesis, lo que culmina con el agradecimiento por la buena disposición a colaborar con el estudio y la obtención del potencial compromiso de acceder a otra sesión en un futuro próximo.

La recogida de datos como producto de la aplicación de entrevistas etnográficas no concluye porque los entrevistados no posean más información; normalmente se pone término a la aplicación de instrumentos porque se acaba el tiempo contemplado para el trabajo de campo, se consideran logrados los objetivos de la etapa, se agotan los recursos o la paciencia del investigador (15). No obstante, es frecuente que las entrevistas etnográficas se realicen en más de una sesión con los mismos entrevistados; esto se aplica en forma especial a las investigaciones que contemplan en su diseño el MSDI.

\section{EL MÉTODO DE LA SECUENCIA DE LA INVESTIGACIÓN DE JAMES SPRADLEY}

El método contempla el proceso científico de conocer personas y grupos durante un período de tiempo utilizando la observación de contexto y las entrevistas como principales técnicas para recopilar información.

Esta estrategia establece cuatro tareas fundamentales para el procesamiento de la información producida por las entrevistas, que son: "El establecimiento de dominios, taxonomía, componentes y temas".

"Los dominios" son categorías de significados culturales que incluyen subcategorías relacionadas semánticamente $(4,16)$. La tarea de determinar dominios se realiza mediante el conocimiento de términos culturales que involucran conceptos relacionados de menor jerarquía, es decir, el dominio posee términos incluidos a través de relaciones semánticas. Para establecer los conceptos de su método, Spradley recomienda no preguntar por el significado de un término para establecer un dominio, sino por el uso del mismo en la cultura. Ejemplo de esta relación se presenta en un estudio de enfermería cultural sobre lactancia materna en mujeres con diabetes. Aquí se establecen dominios que las investigadoras identifican cuando las mujeres responden sobre las prácticas de alimentar a sus hijos. Cuando la pregunta se relacionaba al significado de alimentar al bebé, la respuesta ofrecía una breve referencia a lo que ellas percibían en el acto. Sin embargo, el dominio fue establecido cuando las interrogantes aludían al uso del concepto; de este modo alimentar al bebé recién nacido quedó integrado por términos como: "dar de mamar", "dar la teta", y lo que se debió incorporar con posterioridad, "dar tetina" y "dar el biberón", que también se convirtieron en subcategorías para referirse a la sustitución de la lactancia materna en el uso de alimentar al bebé en muchas mujeres diabéticas como resultado de una serie de complicaciones médicas para producir leche (1).

"Las taxonomías" son construcciones conceptuales que se obtienen mediante el establecimiento de las relaciones semán- 
ticas entre todos los términos incluidos en los dominios. Una taxonomía revela la existencia de subconjuntos de términos relacionados en un todo $(4,5)$. En la investigación realizada por Calvo et al, sobre el significado del respeto y cuidado como dimensiones morales del ejercicio de la enfermería, se presenta la construcción de taxonomías que reflejan las relaciones del dominio con sus respectivos términos incluidos. Como parte del uso "trato con respeto", se desprendieron los términos "tono de la voz", "actitud de interés", "palabras simples" y "buenas respuestas" (17). El establecimiento de taxonomías va generando paulatinamente una estructura adecuada para un buen análisis.

La tercer tarea fundamental del MSDI es "El análisis de componentes"; éste surge del establecimiento de dominios y taxonomías, lo que nos provee un marco para realizar la actividad. El procedimiento se funda sobre una actividad reflexiva que permite comprender el significado de un término en la medida que difiere de los demás; en palabras de Spradley es "conocer el significado de un término dependiendo de lo que no signifi$c a^{\prime \prime}(5)$. Continuando con los ejemplos aportados por el estudio sobre lactancia materna, el entrevistador puede preguntar sobre las formas que tiene una madre de alimentar a su bebé recién nacido; es probable que a la respuesta se agreguen datos adicionales o atributos acerca del término. En el estudio de Flor Sanmiguel y Guerra, una respuesta obtenida fue: ... "le doy teta; es lo único bueno que le puedo ofrecer para que no se me vaya a enfermar, como yo" (1). La información que se puede obtener no está relacionada a una sola taxonomía, es posible identificar más de una relación semántica en el fragmento anterior. El análisis de componentes se presenta como una actividad clarificadora que orienta la representación de la información contenida en las respuestas de los entrevistados $(4,5)$.

"El análisis de temas" representa la última de las tareas fundamentales que requiere el MSDI; contiene una serie de procedimiento que la integran. En esta etapa se intenta describir la cultura observada señalando sus características generales y específicas. El objetivo es identificar los elementos cognitivos que constituyen una cultura, lo que sus integrantes creen que es y reconocen como real. El desarrollo de esta fase se reconoce como una de las más exigentes en cuanto al despliegue de habilidades personales del investigador; se carece de recomendaciones claras para ejecutarla, lo que la convierte en una actividad intuitiva. Spradley contribuye con algunas recomendaciones que mezclan acciones generales a los estudios de corte etnográfico con específicas a esta fase del MSDI, entre las que destacan:

\section{- La inmersión}

- Análisis de componentes vinculados a los dominios

- Identificación de dominios amplios

- Elaboración de mapas conceptuales

- Reconocimiento de temas universales

La "Inmersión" es una acción común en los estudios etnográficos cuya característica es la exposición prolongada en los contextos observados. El investigador pasa tiempo en los escenarios, aplica entrevistas, dedica largas jornadas a escuchar, consigna notas de campo y determina temas culturales. No hay acuerdo en torno a la cantidad de tiempo que demanda la inmersión, pero el investigador debe ser consciente que los periodos son prolongados; no es posible describir la complejidad cultural en un lapso breve de tiempo ni establecer los temas necesarios para cumplir con las aspiraciones de esta etapa (4, 11, 12).

En "El análisis de componentes vinculados a los dominios" el observador se percata de una amplia lista de dominios. Éstos necesitan ser contrastados en el campo para determinar similitudes y diferencias visualizando sus relaciones; solo de esta forma conocemos los temas comunes de la cultura. Otra actividad necesaria en la determinación de temas es "Identificar dominios amplios", lo que se traduce en el establecimiento de dominios que abarcan distintas culturas; esto permite el establecimiento de categorías que trascienden el contexto estudiado y pueden servir de referencia a otras investigaciones a través de acciones de contraste amplio.

La "Elaboración de mapas conceptuales" implica graficar los vínculos identificados entre los dominios. Por último, "El reconocimiento de temas universales" es el común denominador de todas las fases y recomendaciones del MSDI. Se trata, fundamentalmente, de la contrastación de la información obtenida de las técnicas aplicadas en el desarrollo del trabajo de campo. Esta etapa involucra la actividad de identificar similitudes y diferencias entre los términos obtenidos, lo que contribuye a profundizar el conocimiento del escenario, aunque el método sugiere trascender el contexto estudiado y no solo comparar los signos al interior; es necesario reconocer temas que son comunes entre diferentes culturas. Spradley sugiere observar una serie de temas que tienen el potencial de ser identificados en todas las culturas observadas entre los cuales destacan: Conflictos sociales, Las contradicciones culturales, Las técnicas informales de control social, Las estrategias relacionales y El estatus.

"Los conflictos sociales" se alzan como el primer tema universal que puede ser identificado en todos los contextos observados. Reconocer que nuestro escenario posee una experiencia que influye en la formación y el mantenimiento de vínculos entre sus integrantes nos otorga la amplitud de visión para identificar conflictos.

"Las contradicciones culturales" se mencionan como otro tema universal relacionado a las inconsistencias en las creencias, aseveraciones e ideas de muchas culturas. Al respecto se ilustra este tema en el informe de investigación sobre práctica de la atención farmacéutica hospitalaria en Cuba. En ésta se mencionan contradicciones vinculadas a los conceptos de integración y trabajo en equipo promovidos por el enfoque de salud familiar que predomina en la práctica de los profesionales del equipo de salud desde su formación universitaria en este país. En el estudio se describen contradicciones relacionadas al reconocimiento del profesional farmacéutico por parte del res- 
to del equipo de salud, principalmente de médicos y enfermeras, que desconocen la evolución de la profesión farmacéutica y la vinculan con sus orígenes de farmacia clínica de la década de los 60 asociada a una etapa de instrucción académica norteamericana. El estudio menciona una serie de estrategias para superar la desmotivación y los problemas que representa para los farmacéuticos el desconocimiento de su labor en establecimientos de salud en Cuba. Se recurre a conductas mediadoras que intentan difundir las potencialidades de estos profesionales en un intento por evitar el freno a procesos participativos que practican los integrantes del equipo de salud (18).

"Las técnicas informales de control social" son un tercer tema universal indicado por Spradley. Se relaciona con las conductas aprendidas, aceptadas y compartidas por los integrantes de la cultura. Estas ejercen una gran influencia en el comportamiento de las personas y se establecen como prácticas para mantener la cultura dentro de un orden implícito, pero de gran influencia entre los integrantes. En el análisis de Madriz sobre la percepción del crimen en la vida cotidiana de mujeres, se describe como la técnica de control social informal que resultaba de la oposición ciudadana a la concreción de delitos; fue decayendo por el temor de las mujeres a sufrir daños físicos por parte de los criminales (19).

"Las estrategias relacionales" son temas universales que se refieren a las formas en que las personas se vinculan con otros que reconocen como cercanos y las estrategias que despliegan para enfrentarse a los individuos que no conocen $(4,5)$.

"El estatus" se considera vinculado al todo cultural y también a los individuos como integrantes. Está relacionado al prestigio y se destaca como una importante fuente de motivación para la conducta que requiere atención del investigador. En un estudio cultural sobre prácticas de salud de comunidades indígenas atacameñas en el norte de Chile se evidencia la importancia que posee la salud para el mantenimiento del estatus. Los atacameños conceptualizan la salud como un estado en el que la persona puede dedicarse a trabajar la tierra sin cansarse en forma excesiva y sin obstáculos físicos, lo que le permite mantener relaciones sociales armónicas. La enfermedad es considerada una situación que no solo afecta el cuerpo sino también significa una pérdida de estatus que los invade de pesar (20).

"La solución de conflictos" es el último tema universal. Todas las culturas presentan diferentes niveles de conflicto y los atienden de acuerdo con particulares estrategias que el investigador debe ser capaz de identificar. En este punto Spradley es claro en señalar que los informantes no expresan directamente los conflictos; éstos deben ser observados cuidadosamente a través de preguntas descriptivas que nos permiten acceder de forma gradual a datos más directos en torno a los problemas y las estrategias de solución. Preguntar por los problemas entre vecinos nos puede arrojar solo respuestas evasivas; sin embargo una pregunta amplia, ¿cómo es la vida en el barrio?, nos permitiría abrir espacios para conocer detalles que caracterizan a estos temas (5).
Una vez que se adquiere conocimiento del escenario y se reconocen los temas culturales, se recomienda hacer un "resumen del estado del escenario estudiado"; éste representa la última estrategia del análisis de temas. Consiste en la redacción de un resumen con las características más destacadas del contexto a modo de carta de presentación para otras investigaciones que podrían plantearse realizar un estudio en el lugar. De esta forma se establece un documento potencialmente útil como primer acercamiento a la cultura; en éste se incluyen las partes más importantes que otorgan una descripción resumida del contexto (4).

Las fases anteriormente aludidas representan la estructura básica del MSDI; no obstante, por motivos de espacio no es posible desarrollar en detalle cada uno de los aspectos originales que contempla, los cuales son expuestos en los textos legados por James Spradley.

\section{CONSIDERACIONES FINALES}

La estrategia señalada guía al investigador en el proceso de conocer y describir lo que observa, se constituye en consejo práctico de gran valor en el momento de realizar el trabajo de campo. El desarrollo de un estudio etnográfico pareciera descansar en una ausencia de reglas metodológicas; sin embargo, el estilo abierto conlleva algunos riesgos necesarios de contemplar. La ausencia de orientaciones dejaría la recopilación de información en el escenario como un ejercicio exclusivo de investigadores avezados en estudios culturales, y por otro lado, el riesgo que representa para las personas ser observadas por investigadores faltos de formación metodológica que podrían producir daños en los contextos, arriesgando el cierre de éstos para futuros estudios que pretendan profundizar fenómenos de interés científico. EI MSDI otorga esa necesaria guía que cubre amplios aspectos que deben atender las investigaciones culturales. La utilización del método descrito va en armonía con las recomendaciones de Strauss cuando señala las ventajas que representa la utilización de orientaciones para el trabajo de campo que supondría un avance considerable en la clarificación de los problemas de investigación.

El presente texto no pretendió el desarrollo detallado de un método, sino más bien señalar la estructura principal de una de las estrategias cualitativas más importantes y claras para los investigadores de diversas áreas del conocimiento. EI MSDI ha sido utilizado en importantes estudios generados por las ciencias sociales y de salud mostrando su potencial como apoyo al desarrollo de investigaciones que tengan por objetivo conocer los aspectos culturales que se explican desde la cotidianidad de la vida de las personas.

\section{APOYOS Y AGRADECIMIENTOS}

El presente artículo contó con el patrocinio del Convenio de Desempeño UTA-Mineduc de las Humanidades, las Artes y las Ciencias Sociales de la Universidad de Tarapacá. 
Cómo citar este artículo: Garrido, N. El método de James Spradley en la investigación cualitativa. Enfermeria (Montev.). [Internet]. 2017 Oct [citado xxxxx]; 6 37-42. Disponible en: https://doi.org/10.22235/ech.v6iEspecial.1449

\section{REFERENCIAS BIBLIOGRÁFICAS}

1. Sanmiguel F, Guerra A. Modelo teórico con enfoque de enfermería cultural sobre lactancia materna en mujeres con diabetes. Salus [Internet]. 2013 [citado Feb 2017]; 17(1): 3439. Disponible en: http://www.scielo.org.ve/scielo.php?script=sci_arttext \&pid=S1316-71382013000100007\&In$\mathrm{g}=\mathrm{es}$

2. Gallagher M, Rehm R. El papel de los síndromes culturales y los remedios tradicionales mexicanos en la promoción de salud de los niños. Enfermería global [Internet]. 2012 [citado Ene 2017]; 11(27): 1-11. Disponible en: http://scielo.isciii.es/scielo.php?script=sci_arttext\&pi$d=$ S1695-61412012000300001\&lng=es.

3. Giraldo D, Calderón H, Rivera Cristian, Velásquez S, Mesa Heidy. Vivencias de familiares en sala de espera de trabajo de parto. Aquichán [Internet]. 2016 [citado Feb 2017]; 16 (2): 205-218. Disponible en: http:// www.scielo.org.co/scielo.php?script=sci_arttext\&pi$d=$ S1657-59972016000200008\&lng=en.

4. Rodríguez G, Gil J, García E. Metodología de la investigación cualitativa. Málaga: Aljibe, 1996.

5. Spradley J. The Ethnographic Interview. EEUU: Hardcourt, 1979.

6. Gaínza A. La entrevista en profundidad individual. En: Canales M (edit). Metodología de investigación social. Introducción a los oficios. 4 a ed. Santiago de Chile: Lom; 2014. p. 219-263.

7. Valles M. Técnicas cualitativas de investigación social. Reflexión metodológica y práctica profesional. Madrid: Síntesis, 2009.
8. Torruco U, Díaz L, Martínez M, Varela M. La entrevista, recurso flexible y dinámico. Investigación en Educación Médica. [citado Feb 2017]. Disponible en: http://www. redalyc.org/articulo.oa?id=349733228009. Fecha de consulta: 16 de marzo de 2017.

9. Guber R. La etnografía, método, campo y reflexividad. Bogotá: Norma, 2001.

10. Díaz de Rada A. El taller del etnógrafo. Materiales y herramientas de investigación en etnografía. Madrid: UNED, 2011.

11. Taylor S, Bogdan R. Introducción a los métodos cualitativos de investigación. Buenos Aires: Paidós, 1986.

12. Hammersley M, Atkinson, P. Etnografía, métodos de investigación. Barcelona: Paidós, 2009.

13. Téllez A. La investigación antropológica. Alicante: Club Universitario, 2007.

14. Sierra F. Función y sentido de la entrevista cualitativa en investigación social. En: Galindo J. (coord). Técnicas de investigación en sociedad, cultura y comunicación. México: Addison Wesley Longman; 1998. p. 277-346.

15. Goetz J, Lecompte M. Etnografía y diseño cualitativo en investigación educativa. Madrid: Morata, 1988.

16. Tojar C. Investigación cualitativa, comprender y actuar. Madrid: La Muralla, 2006.

17. Calvo M, Ayala Ricardo, Holmqvist Moira, Molina Cecilia. Aspectos axiológico-culturales de la ética y el cuidado. Acta bioeth [Internet]. 2011 [citado Feb 2017]; 17(1): 133141. Disponible en: http://www.scielo.cl/scielo.php?scrip$\mathrm{t}=\mathrm{sci}$ arttext\&pid=S1726-569X2011000100015\&lng=es.

18. Einsen $\mathrm{G}$. La atención primaria en Cuba: el equipo del médico de la familia y el policlínico. Rev Cubana Salud Pública [Internet]. 1996 [citado Mar 2017]; 22(2): 4-5. Disponible en: http://scielo.sld.cu/scielo.php?script=sci_arttext\&pi$d=S 0864-34661996000200003 \& \operatorname{lng}=e s$.

19. Madriz E. A las niñas buenas no les pasa nada malo. México: Siglo veintiuno; 2001.

20. Chamorro P, Tocornal C. Prácticas de salud en las comunidades del Salar de Atacama: Hacia una etnografía médica contemporánea Estudios Atacameños [Internet]. 2005 [citado Mar 2017]: 117-139. Disponible en: http://www. redalyc.org/articulo.oa?id=31503007. Fecha de consulta: 16 de marzo de 2017. 